and shrubs, due to a higher ice volume during a summer insolation maximum. As the authors point out, this last interglacialglacial cycle provides the possibility of examining the hierarchy of controls of past climate variations.

The notable point about the record analysed by Whitlock and Bartlein is its accurate dating. This was done with a good set of 13 dates from carbon-14 and volcanic-ash records. Moreover, although the pollen-zone boundaries have been assigned independently of the marine oxygen isotope stratigraphy (SPECMAP $\delta^{18} \mathrm{O}$, a proxy for global ice volume), their ages match the isotopic boundaries very nicely.

Accurate dating is, in fact, the crux of the matter, for lack of it has been the main handicap in analysis of the high-frequency fluctuations in vegetation history that are often inferred from continental (terrestrial) cores. Vegetation records are the products of interactions between physical, geological and biological systems, and the difficulty is in relating such records to a given climate mechanism. So it is not surprising that, in recent years, terrestrial palaeoecology has lagged behind palaeoceanography and glaciology, fields in which dating is less problematic and climate forcing can be interpreted more easily.

For example, most of the terrestrial cores contain a lot of high-frequency variations during the last glaciation, but the various explanations for such variations were rarely convincing. Only after glaciologists discovered the so-called Dansgaard-Oeschger events (temperate episodes in the glacial period), and palaeoceanographers discovered Heinrich events ${ }^{2}$ (colder events associated with iceberg discharges into the North Atlantic ocean), has it been possible to give a climatic explanation to the fluctuations in vegetation seen in continental cores. These climatic events have now been recognized in pollen and loess cores from Florida ${ }^{3}$ to China ${ }^{4}$. The message here is that we need first to understand the climate forcings before being able to interpret terrestrial data, and that these forcings cannot be discovered independently in the terrestrial cores.

Not least, interest in the abrupt climatic events of the past stems from how they might affect us in the future. The present interglacial period (the Holocene, which commenced only about 10,000 years ago) is being analysed to a finer and finer resolution, largely thanks to the wide availability of radiocarbon dates and the existence of annually laminated sediments. Clearly, however, the present interglacial is not yet finished; moreover, records of the course it has taken are sometimes disturbed by man. By contrast, the previous interglacial, stage $5 \mathrm{e}$, gives us the possibility of testing what happens as a glacial period begins by using climate model simulations ${ }^{5}$ and looking at long cores such as that from Carp Lake ${ }^{1}$. Such cores are rare, however, most of them coming from Europe ${ }^{6-9}$ or South America ${ }^{10}$ and often with a timescale dependent on the marine stratigraphy.

The main findings of Whitlock and Bartlein can be generalized to such cores, as illustrated in Fig. 1 by the pollen diagram of La Grande Pile in eastern France ${ }^{11}$. If we transform this pollen diagram into an index ${ }^{12}$ representing fluctuations between forest and herbaceous vegetation, or into a succession of biomes, the correlation with the global climate is evident for timescales higher than 20,000 years - even if, in this case, the timescale has not been derived independently of the ice-volume timescale. The difference between stages $5 \mathrm{e}$ and $5 \mathrm{a}$ that Whitlock and Bartlein point to can also be seen in this figure. The absence of deciduous forest in stage $5 \mathrm{a}$ is likely to be due to cooler winters, which themselves stem from higher ice volume and a cooler Atlantic Ocean.

We need more long sequences, especially in regions which are highly sensitive to climatic changes (for example, the monsoon regions), and analysis of them with sufficient

resolution to detect abrupt events compatible with a human timescale. The climate mechanisms revealed by these records can then be tested with climatic models, so as to help understand the plausibility of future abrupt changes and their effect on the environment.

Joël Guiot is in the CNRS UA 1152, Faculté de St-Jérôme, F-13397 Marseille cedex 20, France. e-mail:Joel.Guiot@lbhp.u-3mrs.fr

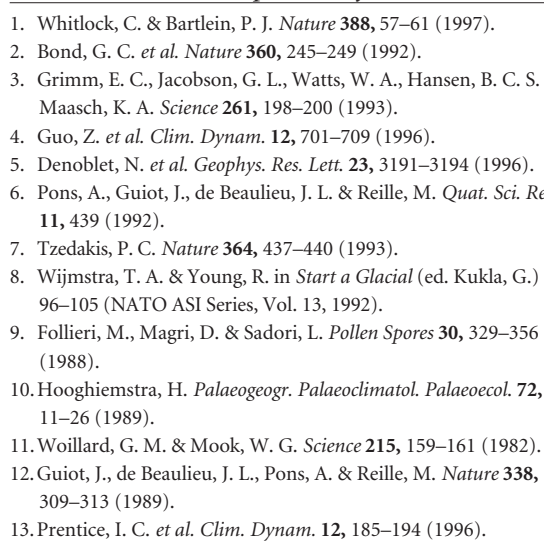

\title{
Environmental engineering
}

The case of the vanishing beaches
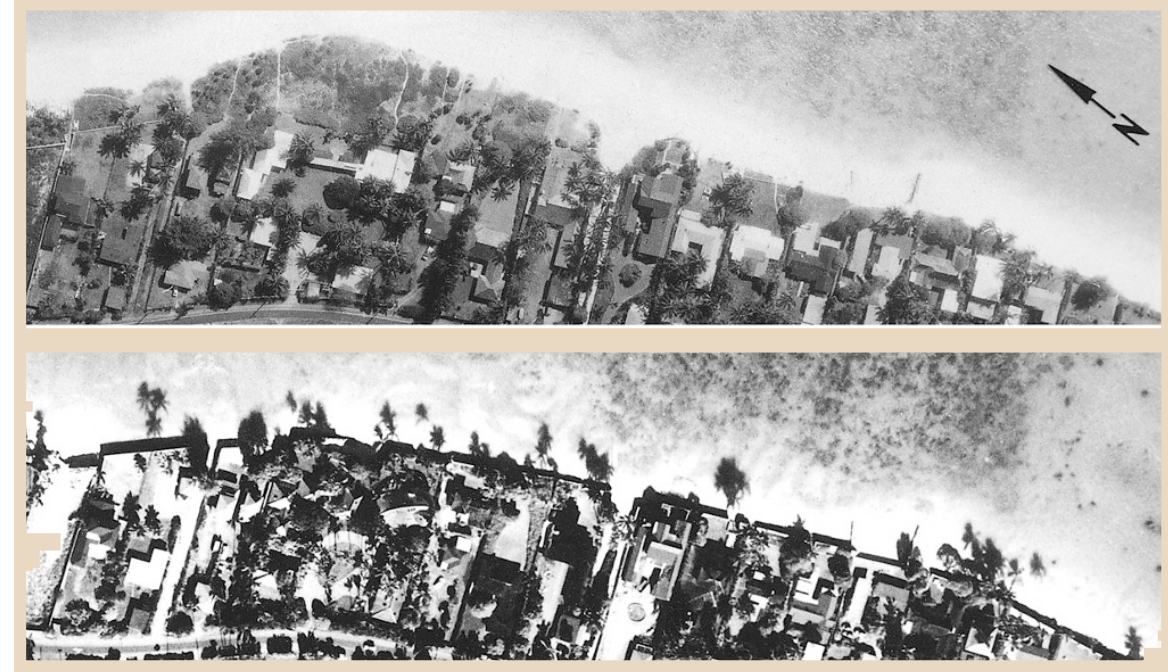

Now (top photograph, 1975) you see it; now (bottom, 1990) you don't. The 'it' is a beach, southeast of Lanikai on the Hawaiian island of Oahu, which disappeared over this period. This and other instances of beach loss or narrowing on Oahu are recorded by Fletcher, Mullane and Richmond in the Journal of Coastal Research (13, 209-215; 1997).

The authors identify coastal armouring structures, built to protect shoreline properties from erosion, as the culprits. The trouble is that armouring concentrates erosional forces on the beach directly in front, which, moreover, also loses the replenishment of sand stores from those locked into shoreline land. In all, the authors estimate that about 24 per cent of the 115 kilometres of Oahu's beaches have been lost or badly affected in this way over the mean (27-year) interval of their study.

Of the consequences discussed by the authors, one is transparent: the possible effects on the Hawaiian economy of a reduction in the number of tourists, who are largely drawn to the islands by the beaches. The 'visitor industry' exceeds by three times that of all others combined in terms of the revenue it delivers to the State of Hawaii.

There are no easy or cheap answers. And as Fletcher and colleagues point out, in a world in which rising sea levels may become an increasing threat, beach loss caused by structures designed to prevent coastal erosion is likely only to increase. Tim Lincoln 